\title{
Appendix B: Transcription conventions
}

Interviews were conducted in Spanish, all translations are mine. The translations attempt to stay close to the original spoken language, rather than revising to idiomatic English. In order to maintain confidentiality in some cases, I give the year and month of interviews throughout but not the exact date.

- /slashes/ are used to indicate overlapping speech between two speakers.

- ... Three dots are used to indicate a pause.

- ---Dashes are used to indicate interrupted speech.

- [ . . ] Brackets with three dots are used to indicate an omission of the original transcript.

- [brackets] are used to indicate an editorial or translation insertion, such as a word that is implied but not actually present in the transcript. 
